\title{
PERCEPCIONES DE INJUSTICIA Y CORRUPCION: EL CASTIGO DE LOS EVASORES
}

\author{
Victoria Giarrizzo* \\ Nicolás Scolnic ${ }^{* *}$
}

enviado: Junio 2011- aceptado: Diciembre 2011

\begin{abstract}
Resumen
Los estudios de evasión suelen focalizarse en el contribuyente como el único sujeto sobre el cual inducir cambios para lograr mejoras en las recaudaciones tributarias. Sin embargo, estudios recientes indican que el comportamiento del Estado determina la conducta de los individuos. Si el Estado es concebido como una institución corrupta, que maneja ineficientemente los recursos, los contribuyentes castigan evadiendo y encuentran justificación fácil a su mal comportamiento fiscal. En ese esquema, la evasión no impacta en la moral de los individuos ni afecta su reputación, y encontrar soluciones se vuelve dificultoso. Este trabajo analiza la evasión como una 'relación tributaria' dinámica entre Estado y sus ciudadanos, con responsabilidades compartidas donde solo cambios simultáneos en ambos actores permitirán reducir la informalidad..
\end{abstract}

Clasificación JEL: H30 - H26 - H24 - K42 - O17

Palabras clave: evasión - contribuyente - estado - responsabilidad

\begin{abstract}
Tax evasion studies tend to focus on the taxpayer as the only subjects on which induce changes for improvements in tax revenues. However, recent studies indicate that State's behavior determines the actions of individuals. If the State is conceived

\footnotetext{
*Facultad de Ciencias Económicas, Universidad de Buenos Aires, e-mail: vgiarrizzo@gmail.com

** Facultad de Ciencias Económicas, Universidad de Buenos Aires, e- mail: nscolnic@gmail.com
} 
as a corrupt institution that manages the resources inefficiently, taxpayers punish with avoidance and find easy justification to his bad fiscal behavior. In this scheme, evasion does not impact on the morale of individuals or affect their reputation, and finding solutions becomes difficult. This paper analyzes the avoidance as a dynamic 'tax relationship' between the State and its citizens with shared responsibilities where only simultaneous changes in both actors will reduce informality.

JEL Classification: H30 - H26 - H24 - K42 - O17

Keywords: evasion - taxpayer - state - responsibility

\section{INTRODUCCION}

La evasión tributaria es un problema que afecta a la mayoría de los gobiernos del mundo. En mayor o menor medida, todos los países cuentan con determinados niveles de informalidad, que obligan a sus gobiernos a idear mecanismos para reducir la economía en negro, incrementar su recaudación y resolver las distorsiones e inequidades que provoca el "no pago de impuestos" de algunos ciudadanos.

La Argentina no escapa a esta generalidad. Aunque en los últimos años se fueron mejorando los métodos de recaudación a partir del diseño de mejores estrategias de comunicación, del desarrollo de tecnologías más eficientes, y de la intensificación de los controles, la evasión continúa siendo un tema grave que genera importantes distorsiones entre quienes pagan y no sus tributos, y alienta a sobre-presionar a los contribuyentes que cumplen con sus obligaciones tributarias. Los datos del Ministerio de Economía de la Argentina dan evidencia de la mayor presión tributaria aplicada en los últimos años: si en 2001 la recaudación tributaria nacional era equivalente a 17,3\% del Producto Interno Bruto (PIB), en 2010 equivalía a $28,9 \%$ del PIB, lo que significa un aumento de 11,6 puntos solo en la presión tributaria nacional. Ese aumento, lejos de ser de vaga importancia, tiene consecuencias en términos de equidad y competitividad.

Un análisis ligero del incremento en el ratio recaudación/PIB, permite intuir que la mayor presión tributaria no fue correspondida con una disminución proporcional en los niveles de informalidad. Esto deja entrever la existencia de un esquema tributario distorsivo, donde unos "pagan todo", otros "pagan algo" y otros directamente "no pagan". En ese esquema, quienes cumplen con sus obli- 
gaciones se ven exigidos a abonar proporciones crecientes de su ingreso. Esta situación obliga a repensar cuestiones tan básicas como: ¿por qué en una sociedad un porcentaje tan alto de sus ciudadanos incumplen sus obligaciones tributarias?

La evasión tiene causas muy heterogéneas que varían de país en país, y dentro de una misma economía se presentan diferencias sustanciales según estratos de ingresos, niveles de educación, edad, género, ubicación geográfica o características culturales de la población. Sin embargo, numerosos estudios vienen demostrando que la evasión se presenta con mayor frecuencia en países con baja moral tributaria, y en el caso puntual de la Argentina, se suceden dos hechos particulares. Por un lado, un porcentaje elevado de la población o no tiene moral tributaria o su moral tributaria es débil, lo que genera una propensión natural a evadir; y por otro, incluso entre sectores de la población con moral tributaria muy fuerte, se observa una predisposición elevada a no contribuir lo que corresponde ('moralistas evasores' en Giarrizzo-Sívori, 2010). Esto nos coloca frente a otras dos incógnitas: 1) ¿Por qué es tan significante la ausencia de moral tributaria en algunos segmentos poblacionales? y 2) ¿por qué una persona con moral tributaria está dispuesta a evadir?

La hipótesis que exploraremos en este trabajo es que mientras en los segmentos poblacionales que no tienen moral tributaria la evasión es una práctica habitual, que se extiende en su máxima dimensión posible en la medida que los controles (o la insuficiencia de ellos) lo permiten, en los sectores poblacionales donde sí existe algún grado de moral tributaria (ya sea fuerte o débil) la evasión está estrechamente relacionada con la percepción de injusticia y corrupción que tienen los ciudadanos sobre el Estado. La sensación de que existen ineficiencias importantes en el direccionamiento de los recursos tributarios alienta a evadir. Como patrón general, estudios empíricos permiten detectar que en los segmentos de moral tributaria fuerte y débil, los mismos evasores encuentran justificación a su conducta de 'evadir', presentándola como una especie de castigo hacia el Estado porque: a) consideran que coexisten en él elevados niveles de corrupción, b) presumen que esa corrupción deteriora su poder adquisitivo; y c) porque perciben que los impuestos que pagan no siempre son justos o que la distribución de los recursos fiscales no es la adecuada. Esto se observa principalmente en el segmento de la población que, a pesar de contar con moral tributaria fuerte, se muestra predispuesta a evadir ('moralistas evasores').

Efectivamente, es común encontrar en la Argentina ciudadanos con elevada moral tributaria predispuestos a evadir, que justifican ese acto que ellos mismos 
consideran inadecuado, en los altos niveles de corrupción estatal o en la existencia de inequidades en las tasas impositivas que se cobran. Sin embargo, de acuerdo con la Encuesta de Percepción Fiscal (EPF) realizada en el marco de esta investigación, también la ausencia de moral tributaria parece estar relacionada a la percepción de niveles elevados de corrupción, donde el individuo actúa emulando a quienes lo gobiernan. Este comportamiento se incrementa cuando la población percibe dificultades de ingresos, no solo porque adopta a la evasión como una forma de supervivencia, sino porque encuentra en las ineficiencias del Estado las causas de sus problemas. En síntesis, incluso asumiendo que existen grupos sociales con diferentes grados de moral tributaria, la existencia de corrupción e ineficiencias en el sector público afecta de alguna manera a cada una de las categorías.

Lo que se intentará demostrar en este estudio es que posiblemente los mismos factores que explican que evada un individuo que manifiesta contar con moral tributaria fuerte, son los que explican que haya una porción elevada de personas en la Argentina sin 'moral tributaria'. Y estos factores tienen que ver con la percepción de injusticia y corrupción sobre la clase gobernante, factores cuya corrección escapa a los organismos de control fiscal (AFIP en la Argentina) y dependen en cambio, de la voluntad institucional del gobierno de reducir los niveles de corrupción entre sus funcionarios.

La relación entre evasión y percepciones de injusticia, ineficiencias y corrupción parece muy estrecha en la argentina. En la medida que la gente percibe niveles de corrupción elevados, la evasión deja de ser considerada una conducta delictiva. Sin embargo, este tipo de comportamientos puede llevar a esquemas delicados, donde el individuo encuentra en la corrupción la excusa o justificación a su incumplimiento fiscal. Es decir, la causalidad se invierte. El individuo evade porque persigue un fin económico (en general, cuando un individuo evade es para incrementar su ingreso disponible), pero al momento de justificar su conducta, utiliza como argumento la corrupción e injusticia en el sistema tributario.

Esto lleva a una conclusión obvia: reducir la evasión en países con elevados niveles de corrupción es dificultoso, justamente porque la no decisión de contribuir es la forma en que los individuos manifiestan su desacuerdo con ese sistema que explícita o implícitamente consideran distorsivo. En otras palabras, un importante porcentaje de los contribuyentes consideran el no pago de impuestos como un castigo al Estado corrupto. Y revelan que, aún si tuvieran el estándar de vida deseado, tampoco pagarían sus impuestos, pero sí lo harían si hubiera menos corrupción. De esa forma, y sobre la base de un Estado con altos niveles de corrup- 
ción, es muy difícil construir una moral tributaria sólida y consistente, elemento indispensable para reducir la economía informal.

El objetivo de este trabajo será explorar el vínculo entre evasión, percepciones de injusticia, de ineficiencias y corrupción, para establecer qué tipo de causalidad existe, y hasta dónde la evasión es consecuencia del comportamiento del Estado. Eso invierte de alguna manera el enfoque tradicional, donde se presume que el problema de la evasión está en las conductas de los individuos, y se pone la mirada en la responsabilidad de un Estado que a través de su comportamiento, alienta la evasión. Así, exploraremos un enfoque de responsabilidad compartida entre el Estrado y la población que permite evaluar la incidencia de ese Estado en el comportamiento fiscal y las limitaciones para combatir el problema en la medida que el Estado no dé señales de fortaleza institucional vinculada al buen manejo de los fondos públicos.

\section{LA EVASION Y SUS NUEVOS METODOS DE ESTUDIO}

Durante siglos la evasión tributaria fue tema de atención y preocupación entre los gobernantes necesitados de recursos monetarios para hacer frente a sus gastos. Y la economía no se mantuvo ajena a ese interés: desde su conformación como ciencia, la evasión, sus causas y los posibles métodos para combatirla fueron temas abordados intensamente por la literatura científica. Pero la forma en que se manifestó ese abordaje sufrió modificaciones en el tiempo. De los primeros trabajos basados en la simple observación del problema, se pasó en la década del '60 a la elaboración de modelos teóricos que buscaban una explicación más universal y objetiva de la evasión.

Sin embargo, los resultados no conformaron. Ante la ausencia de respuestas únicas y frente a las dificultades de los gobiernos para combatir la evasión en base a los resultados teóricos obtenidos, se abrieron nuevas ramas de estudio que fueron modificando por un lado, el 'cómo' estudiar y analizar el problema de la evasión y por otro, el 'qué' variables analizar al abordar su estudio. En el método de estudio (el 'cómo') se introdujeron dos formas de análisis con resultados prometedores: la experimentación y el empirismo promovidos a partir de experimentos de laboratorio y la elaboración de encuestas que buscaban entender el comportamiento fiscal de los individuos. En el qué estudiar, en tanto, la novedad (a partir de la década del '80 principalmente) provino de la incorporación de un conjunto de variables 
subjetivas con alta capacidad explicativa de diversos aspectos relacionados con el pago o no pago de impuestos.

Estos métodos posibilitaron abrir más preguntas y encontrar más respuestas, pero todos tenían una característica: el objeto de estudio central de la evasión, era el contribuyente y en pocas oportunidades se ponía la mirada en la responsabilidad del Estado en el acto de evadir. Analizar la responsabilidad del Estado implica revertir la causalidad del fenómeno de la evasión y dar lugar a una nueva hipótesis: ¿y si el comportamiento de los contribuyentes es un reflejo del comportamiento del Estado?

\section{I.1. Hacia la constitución de una teoría}

La evasión fiscal comienza a estudiarse en forma sistemática a fines de la década del ' 60 con el desarrollo de un conjunto de modelos teóricos, que más allá de los resultados que arrojaban, permitieron colocar el tema en la agenda de los economistas. Hasta ese momento, la mayoría de los trabajos sobre evasión eran acotados, con bajo grado de modelización. En algunos casos sustentados en relevamientos empíricos aislados realizados en pequeñas ciudades y de los cuales se desprendían una serie de relaciones insuficientes para construir una teoría que permitiera abordar el problema de la informalidad desde el plano teórico. Lo curioso, sin embargo, es que esos estudios ya dejaban entrever que existían determinantes subjetivos en el acto de evadir (factores dejados de lado en los primeros trabajos teóricos). Un trabajo relevante, fue el de Schwartz y Orleans (1967), donde se demostraba que la llamada a conciencia fiscal podía resultar más efectiva que las sanciones legales.

A partir de la década del '70, gran cantidad de investigadores se lanzaron a buscar respuestas a la conducta fiscal de la población. Si bien inicialmente esos estudios se focalizaron en los fundamentos objetivos de la evasión, la novedad fue que el problema del incumplimiento tributario comenzó a ser analizado desde una perspectiva teórica, utilizando buena parte del instrumental analítico que por esas épocas incorporaba la ciencia económica. Así surgieron numerosos modelos basados en un conjunto de parámetros objetivos, que analizaban las causas de la evasión y simulaban mecanismos para reducirla. Uno de los trabajos más populares fue el de Allingham y Sandmo (1972), quienes desarrollan un modelo que permite analizar a partir del grado de aversión al riesgo de los individuos, como juegan ciertas variables objetivas como la tasa de penalidad y la probabilidad de inspección en la decisión de pago. 
Los principales trabajos por esos años, se focalizaron en los métodos de control ejercidos desde el Estado. Es decir, la solución a la evasión vendría a partir de la manipulación de variables controladas por el Estado (controles, multas y tasas principalmente) y sus resultados quedarían plasmados en la función de utilidad definida individual y socialmente a partir del trabajo de Von Neumann y Morgenstern (1944). En síntesis, esta primera fase modelos microeconómicos explicaban la actitud del contribuyente partiendo de la premisa de que las únicas variables que influían en su comportamiento fiscal eran factores objetivos donde toda la responsabilidad era del contribuyente. La solución entonces, vendría a partir de un cambio en su actitud. La función del Estado en este esquema, era inducir ese cambio a partir de la aplicación de penalidades y controles.

Con el apoyo teórico de estos trabajos, los Estados fueron ideando e implementando medidas para disminuir los niveles de evasión. Sin embargo, los resultados no fueron los esperados: el tamaño de economía informal en la mayoría de los países que lideraban en el campo de estudios teóricos de la evasión, fue incrementándose a través de las décadas

Cuadro 1 - Evolución del tamaño de la economía en negro (en $\%$ del PIB)

\begin{tabular}{lccccccc}
\hline & $\mathbf{1 9 7 0 - 1 9 7 5}$ & $\mathbf{1 9 7 6 - 1 9 8 5}$ & $\mathbf{1 9 8 6 - 1 9 9 0}$ & $\mathbf{1 9 9 4}$ & $\mathbf{1 9 9 6}$ & $\mathbf{1 9 9 8}$ & $\mathbf{2 0 0 2 - 2 0 0 3}$ \\
\hline Alemania & 4,5 & 8,5 & 11,3 & 13,1 & 14,5 & 14,8 & 16,8 \\
Italia & 11,3 & 15,4 & 21,3 & 25,8 & 27,0 & 27,8 & 26,2 \\
Inglaterra & 4,3 & 8,2 & 9,7 & 12,4 & 13,1 & 13,0 & 12,3 \\
Canadá & 5,1 & 7,6 & 12,0 & 14,6 & 15,1 & 15,0 & 15,4 \\
Estados Unidos & 3,5 & 5,0 & 6,2 & 9,4 & 8,8 & 8,9 & 8,6 \\
\hline
\end{tabular}

Fuente: IFO (2009)

Las estadísticas sobre evasión y economía informal que se iban conociendo, sugerían preguntas para las cuales no había respuestas claras: ¿por qué la evasión crecía a pesar de los controles? ¿por qué en un país donde la probabilidad de inspección es más alta que otro su tasa de evasión representa más del doble? O más raro aún, ¿por qué en sociedades donde la penalidad por evadir es baja persisten bajos índices de evasión o donde la penalidad es alta la evasión también lo es? Los modelos tradicionales y la teoría clásica resultaron, solas, insuficientes para comprender y dar un diagnóstico acertado de la evasión que posibilite combatirla. 


\section{I.2. Consolidación del Enfoque Suave del cumplimiento fiscal}

La insuficiencia de las explicaciones aportadas por la economía sobre los motivos del comportamiento de los contribuyentes, alentó a diferentes ciencias a avanzar en el estudio de otros parámetros explicativos de la evasión. Lo moral, lo social, la culpa, la reputación social, la corrupción estatal, comenzaron a aparecer como causas relevantes que hasta el momento habían sido escasamente exploradas, y la economía no ignoró esa posible vía de abordaje. El análisis económico tradicional de la evasión comenzaba así a ser útilmente complementado por otros enfoques.

Pero otra novedad se daba por estos tiempos. No solamente habían sido incorporados nuevos parámetros al estudio de las causas de la evasión, sino que el método también había encontrado una alternativa o, más bien, un buen complemento. Con el avance de la experimentación en estas otras ramas de la economía y la psicología, se consolidó lo que se denominó Enfoque Suave del cumplimiento fiscal. Este enfoque buscó explicar cómo las características de los contribuyentes, sus percepciones, su moral o su sensación de equidad, se sumaba a las cuestiones analizadas tradicionalmente (tasas impositivas, probabilidades de inspección y sanciones) como factores explicativos de la evasión. El enfoque concentraba su explicación en la actitud del contribuyente hacia el Estado, el sistema y hacia otros contribuyentes El conjunto de interacciones son las que terminan de definir si el ciudadano delinque o no. Su manera de vincularse con sus pares y las instituciones moldean su comportamiento de manera decisiva. Es por esto, que otras ciencias sociales comenzaron a ser fundamentales para entender este complejo entretejido de relaciones que involucra la decisión de pagar o evadir. De aquellos estudios surgía que cuando la postura sobre las políticas de Estado era en general positiva, resultaba probable que se verifique un mayor nivel de cumplimiento (Cullis y Lewis, 1997)

En este contexto, los estudios empíricos en un principio y los experimentales y de simulación, en una segunda fase, comenzaron a tomar protagonismo a partir de 1980 como campos que brindarían nuevas respuestas a interrogantes sobre el comportamiento fiscal. Junto a ello, la incidencia de variables subjetivas como lo moral, la reputación social, o las percepciones de eficiencia, justicia y equidad también ganaron terreno como factores que no podían pasarse por alto para entender las causas íntimas que guían el comportamiento de los contribuyentes. La mayoría de los estudios de este tipo fueron realizados inicialmente en Estados Unidos y Europa. Latinoamérica, particularmente, comenzó tardíamente a focalizarse en este tipo de estudios con lo cual lleva la retaguardia frente a otros 
países. Casualmente o causalmente, el retraso en introducir estudios empíricos y experimentales resultó una constante en aquellos países menos desarrollados donde existe un porcentaje importante de economía en negro y una relación negativa entre moral tributaria y economía informal (Ver Weck 1983, Tanzi 2000, Togler, 2005a).

Los primeros resultados que arrojaban los relevamientos empíricos entre los contribuyentes no solo contradecían muchos resultados de los modelos clásicos, sino que ponían en evidencia las limitaciones de la teoría económica tradicional para explicar la naturaleza de la evasión fiscal. Si bien desde fines de la década del '60 se fueron sucediendo una serie de estudios sobre evasión elaborados en base a encuestas a contribuyentes, sólo fueron considerados por la comunidad económica más de una década después. Estos trabajos buscaban captar la naturaleza de los sujetos y entender por qué actuaban de cierta manera, cuáles eran sus percepciones, sensaciones y motivos intrínsecos que determinaban su propensión a pagar o evadir. En diferentes partes del mundo y con diferentes propósitos, estudios con esta metodología fueron incrementándose, permitiendo detectar un nuevo conjunto de causas para explicar la evasión no consideradas hasta el momento.

Un tema que particularmente despertó el interés de muchos investigadores fue la percepción de tasa justa como determinante de la decisión de pagar. En Estados Unidos, un estudio comparativo realizado durante la década del ' 70 con dos encuestas idénticas pero con un intervalo de cinco años demostró que los sujetos descontentos con las tasas que debían pagar tendían a evadir más que la media (Mason y Calvin, 1978). Waneryd y Walerud (1982) demostraron esto mismo en los contribuyentes suecos. En 1997, Bosco y Mittone desarrollan una interesante reflexión sobre ese punto. Cuando el individuo deja de considerar que lo que está pagando está englobado en un marco de "tasa justa", este concepto desaparece y se entra en una nueva dimensión de análisis: comienza el juego del castigo al Estado. Mientras menos justa sea considerada la tasa, mayor será el castigo, que se manifiesta mediante una mala conducta tributaria. Los autores concluyen que el nivel de evasión dependerá negativamente del juicio subjetivo sobre el nivel promedio de contribución y positivamente de la creencia subjetiva de proporción actual de evasores. Estos estudios sentaron bases para comenzar a estudiar la responsabilidad directa del Estado en la conducta evasora. 


\section{I.3. Cambio en el objeto de estudio: del contribuyente al Estado}

Una serie de trabajos realizados a partir de los ' 90 ya permitían entrever una nueva óptica de análisis. Si se toma en cuenta que existe una determinada penalidad como la que consideran Bosco y Mittone (1997), quiere decir que la primera acción, la base de todo el proceso del pago de impuestos, surge desde el Estado. Por lo tanto, no se pueden encontrar soluciones a largo plazo y definitivas a la evasión sino se hace una modificación en el objeto de estudio. Estas conclusiones ya eran anunciadas desde la psicología social en la década del 80'. Efectivamente, Ajzen y Fishbein, (1980) y Lewis, (1982) indicaban que la falta de cumplimiento fiscal es uno de los únicos mecanismos por el cual el sujeto puede penalizar a un Estado, sistema o Gobierno que no cumple con sus objetivos. Es decir, cuando un individuo evade posiblemente esté enviando un mensaje de disconformidad, cuyo descifrado puede resultar útil a la hora de entender la conducta fiscal y revertirla cuando no es la esperada por los gobiernos.

Uno de los primeros hechos que comenzaron a revelar las encuestas fue la forma en que los individuos juzgan la eficiencia del Estado en el cobro de impuestos y como la aplicación del gasto incidía en la predisposición a pagar. Así, por ejemplo, sociedades con alta evasión coincidían con que los individuos revelaban elevadas percepciones de corrupción por parte del Estado o ineficiencias e injusticias en la forma de recaudar y/o gastar. Los deseos de comprender un poco más los efectos de la corrupción y la sensación de injusticia sobre el cumplimiento tributario, llevó a la realización de una serie de trabajos en países desarrollados y su posterior comparación con resultados en países en desarrollo, donde la relación corrupción-incumplimiento era positiva y más elevada. Una manera interesante de abordar el tema fue el reciente trabajo de Abdul-Gafaru Abdulai (2009) quien sobre datos recolectados en Ghana elaboró un posible camino de combate de corrupción a partir de la exitosa experiencia de otros países en vías en desarrollo o que alcanzaron un estatus superior recién en los últimos años. Fundamentalmente toma como ejemplo Singapur y Hong Hong. Los principales resultados indican que para controlar de manera sostenida la corrupción se requiere una demostración consistente de compromiso genuino desde los niveles políticos más altos hacia la erradicación total de la amenaza. Esa es la única manera de lograrlo. Para eso, debe haber primero una conciencia social generalizada entre todas las partes que están inmersas en los procesos políticos. En segundo lugar, debe haber organismos anticorrupción independientes que trabajen eficientemente para lograr generar incentivos en la gente para comportarse correctamente. A su vez estas entidades deben ser eficaces para encontrar a aquellos que optan por delinquir. Los incentivos y los castigos son claves en el éxito del propósito. 
La relación estrecha entre percepciones de corrupción, confianza en el gobierno y evaluación de las políticas públicas en general comienza a quedar cada vez más en evidencia a partir de una sucesión de trabajos empíricos realizados en diferentes países. Entre ellos, Torgler y Martinez (2005) concluyeron que la mayor confianza y la adhesión a políticas públicas realizadas por el gobierno español permitieron reducir la evasión fiscal del $60 \%$ de finales de 1970 hasta el $10 \%$ a comienzos del 2000. En ese mismo año el primero de los autores realizó un trabajo compilador de los estudios en relación a la moral tributaria en América Latina y arriba a la conclusión de que a mayor satisfacción de los funcionarios públicos, la moral fiscal es mayor. En ese mismo sentido, un estudio empírico en 30 países (Picur y Riahi-Belkaoui, 2006), demostró que el cumplimiento tributario está relacionado negativamente a los niveles de burocracia y positivamente al éxito en el control de la corrupción. O visto de de otro modo, mientras mayor sea el nivel de burocracia estatal y mayor sea la corrupción, los niveles de incumplimiento fiscal serán mayores. Así, generar un marco donde el actuar de los funcionarios públicos y el gobierno en general sean bien vistos, parecía ser esencial para lograr una mayor propensión a contribuir.

Las manifestaciones de injusticia sobre la provisión de bienes públicos constituyen otro factor clave que explica el comportamiento evasor. Bosco y Mittone (1997) afirman, a partir de una serie de juegos de simulación realizados en la Universidad de Trento en Italia, que la redistribución de los ingresos es clave en el comportamiento de los ciudadanos. Ya Alm et al (1992) habían llegado a la conclusión de que la provisión de un bien público financiada con impuestos reduce el nivel de evasión e incluso que la reducción en la evasión sería mayor si los contribuyentes decidieran el destino de sus impuestos. En España, Fatás y Roig (2004) logran los mismos resultados concluyendo por medio de un trabajo experimental con 80 alumnos de la Universidad de Valencia, que se logra un menor nivel de evasión cuando la distribución de los recursos es más igualitaria.

Lo diferente de este conjunto de trabajos es el cambio en el objeto de estudio, siendo el Estado el impulsor o responsable primario de la conducta de los contribuyentes. La carga fiscal, la falta de honestidad, el alto nivel de burocracia, la ineficiencia en el manejo de recursos y la corrupción, constituyen factores relevantes al momento de explicar los motivos por los cuales se evade impositivamente. Mientras más injusto sea considerado el sistema o mientras más corrupción se perciba, mayor será la propensión a evadir. 


\section{PERCEPCIONES DETERMINANTES EN LA MORAL TRIBUTARIA Y LA CONDUCTA FISCAL}

Si hay un punto al cual adhieren la mayoría de los trabajos sobre comportamiento tributario que abordaron los determinantes subjetivos, es que los valores morales constituyen un elemento necesario para reducir la evasión. Numerosos estudios realizados inicialmente en economías desarrolladas y más recientemente en países en desarrollo, arrojan evidencia sobre la incidencia de la moral tributaria en la conducta fiscal de los individuos. Sin moral, el cumplimiento tributario queda en manos de las penalidades y controles, incrementando significativamente el costo de administración fiscal y sin asegurar resultados positivos, ya que no se estarían atacando la raíz del problema, es decir, los motivos por los cuales la moral tributaria es baja. Pero al mismo tiempo, estudios recientes muestran que si bien la moral tributaria es condición necesaria para tener bajos niveles de evasión, no es condición suficiente. En otras palabras, la moral tributaria puede coexistir con la conducta evasora (Giarrizzo y Sivori, 2010) cuando se conjugan una serie de condiciones como las que parecen ocurrir en Argentina.

Esto nos lleva a discutir dos puntos planteados en la introducción. Por un lado, por qué hay segmentos poblacionales con baja moral tributaria en el país, y por otro, por qué aún cuando existe moral tributaria, un individuo puede estar igualmente dispuesto a evadir. Para ser más específicos, lo que estamos buscando es una explicación a por qué los niveles de evasión en la Argentina son tan elevados, pero enmarcada en la cuestión moral, tanto cuando la moral está presente en los individuos, como cuando no lo está.

En la búsqueda de respuestas, surge un elemento cuya incidencia parece ser elevada: las percepciones que tiene la población de injusticia y corrupción en el Gobierno, y la apreciación subjetiva de cómo el gobierno maneja los recursos tributarios son elementos determinantes en el comportamiento tributario. Se intuye que esos factores alientan simultáneamente a: 1) el deterioro o pérdida de la moral tributaria; 2) la evasión como forma de expresar el descontento con esa situación; y 3) la evasión sin culpa usando como argumento la falta de honestidad de las autoridades.

Diversos trabajos sobre evasión y sus causas ya advierten sobre la importancia de las percepciones de injusticia y corrupción en la predisposición al pago de los individuos (Mason y Calvin, 1978; Waneryd y Walerud, 1982; Mittone 1997, 2002a, 2002b, Torgler y Martinez, 2005; Picur y Riahi-Belkaoui, 2006; Abdulai, 
2009). Para analizar esa relación (evasión/percepciones de corrupción; evasión/ percepciones de injustica y evasión/percepciones de deficiencias en la provisión de bienes públicos) se llevó adelante una Encuesta de Percepción Fiscal (EPF), tramo II. El relevamiento se realizó entre octubre y diciembre de 2010 como continuación del tramo I de la EPF (2009). El segundo tramo de la EPF se ejecutó en Capital Federal, Gran Buenos Aires y nueve ciudades del interior del país seleccionadas según un conjunto de criterios metodológicos, donde el parámetro a considerar fue el tamaño poblacional. En total se relevaron 3.300 casos efectivos.

El objetivo de la encuesta fue evaluar la moral tributaria de los individuos, pero poniendo puntual énfasis en la incidencia de las percepciones de corrupción en el gobierno, en las percepciones de justicia y equidad en el sistema tributario y en las apreciaciones de la población sobre la eficiencia del Estado en la provisión de bienes y servicios públicos. La EPF tramo II se realizó entre personas de ambos sexos, mayores de 18 años, de diferentes estratos sociales y los resultados obtenidos parecen confirmar la incidencia de ciertas motivaciones intrínsecas en la decisión de cumplir o no con las obligaciones fiscales. En otras palabras, en contextos donde las percepciones de corrupción, ineficiencias en la administración de recursos públicos e injusticias en la asignación de fondos son elevadas, la evasión como comportamiento tributario en algunos grupos de individuos podría ser entendida como un mecanismo a través del cual los contribuyentes castigan deliberadamente al Estado (Evasores Justicieros), en otros en tanto, no es un castigo deliberado pero sí un espejo del propio funcionamiento de las instituciones.

La corrupción en las instituciones públicas es un problema extendido en la mayoría de los países del mundo, pero principalmente en naciones menos desarrollas, donde la causalidad circular entre corrupción y desarrollo económico dificulta encontrar solución al problema y en cambio, lo perpetúan. La corrupción debilita la confianza de la población, sus valores morales, y, en la medida que los recursos que debieran destinarse a la provisión de servicios sociales son utilizados discrecionalmente para otros fines, deteriora la calidad de vida de las personas. A su vez, la corrupción excluye a los sectores pobres y marginados de las decisiones que afectan sus vidas y puede causar un estado de resignación y apatía, debilitando el compromiso de los ciudadanos con su sociedad (Transparencia Internacional, Informe anual 2008, p.25).

La percepción de corrupción en la Argentina ha sido históricamente elevada, y relevamientos que instituciones diversas vienen realizando en el país confirman que el problema continúa en dimensiones graves. Según el Indice de Percep- 
ción de la Corrupción (IPC) que elabora la Organización Transparencia Internacional, la Argentina se ubica entre los países de mayor percepción de corrupción estatal en el mundo. El IPC mide el nivel de percepción de corrupción del sector público, de acuerdo a la opinión de diversos analistas del país y empresarios. El índice asigna una puntuación a cada país según una escala de cero (muy corrupto) a diez (sin corrupción). En la medición presentada en septiembre de 2009 con datos correspondientes a 2008, la Argentina se ubicó en el puesto 109, con un puntaje de apenas 2,9, muy lejos de naciones como Dinamarca, Nueva Zelanda y Suecia que compartían la puntuación más alta $(9,3$ puntos en una escala donde el máximo es 10).

A nivel local, la Escuela de Gobierno de la Universidad Torcuato Di Tella elabora mensualmente un Indice de Confianza en el Gobierno (ICG) en base a preguntas relacionadas con la percepción que tiene la población sobre: a) la corrupción en el gobierno; b) la eficiencia en el manejo del gasto público; c) la búsqueda del bien común en las políticas públicas que se implementan y la forma en que se asigna el gasto. Sobre una escala de 0 a 5 , donde 0 marca desconfianza total y 5 confianza total, la Argentina alcanzó un valor de 2,42 puntos (datos a marzo 2010). A su vez, según este indicador, solo 51\% de la población revela que los funcionarios del gobierno son honestos ( $49 \%$ dice que no lo son), $43 \%$ dice que se maneja el gasto con al menos cierto grado de eficiencia, en tanto solo el $41 \%$ opinó que se gobierna en beneficio del interés general. Es decir, los niveles de percepción de corrupción son relativamente altos y la percepción de manejo eficiente del gasto, baja.

La pregunta que surge a partir del análisis de esos datos, es cómo incide esa percepción de corrupción e injusticia en la conducta fiscal de los contribuyentes. La literatura económica y la psicología social han analizado extensamente este tipo de vínculos en los últimos 30 años. Lewis (1982) sostiene que es posible que la evasión fiscal sea el canal a través del cual los contribuyentes expresan su antipatía. Así, la relación entre los contribuyentes y el Estado puede ser concebida como una especie de contrato psicológico, que involucra fuertes vínculos emocionales y lealtades (Feld \& Frey, 2002a). La corrupción, generalmente, acarrea una sensación de frustración al ciudadano, minando su moral fiscal (ver Torgler, 2005).

Los resultados de la EPF, parecen confirmar estas conclusiones: las percepciones de corrupción en la Argentina inciden en las decisiones de comportamiento fiscal de los individuos. Y, a la luz de los datos, si la percepción de corrupción se redujera, el público revela estar dispuesto a incrementar sus niveles de cumpli- 
miento tributario. La pregunta que se utilizó en la EPF tramo II realizada en Capital Federal, Gran Buenos Aires y nueve ciudades del interior del país, para evaluar esa relación fue la siguiente:

Si hubiera menos corrupción en el gobierno, ¿pagaría todos sus impuestos?

Las respuestas de los individuos indicarían que, una menor percepción de corrupción en el gobierno alentaría a los contribuyentes a un mayor pago de impuestos. Esta relación es más resaltada entre los individuos que tienen moral tributaria fuerte $(65,3 \%$ de la población relevada), mientras que se reduce entre quienes tienen moral tributaria débil o nula ${ }^{1}$, aunque esa reducción no parece tan significativa. Por ejemplo:

- Entre quienes tienen moral tributaria fuerte: el 71,3\% asegura que si hubiera menos corrupción en el Estado pagaría todos sus impuestos.

- Entre quienes tienen moral tributaria débil: el 50,3\% asegura que si hubiera menos corrupción en el Estado pagaría todos sus impuestos.

- Entre quienes no tienen moral tributaria: el 54,6\% asegura que si hubiera menos corrupción en el Estado pagaría todos sus impuestos.

Lo llamativo, es que aún entre quienes no tienen moral tributaria, es decir, quienes no consideran como algo malo no pagar impuestos, se revela una mayor predisposición a pagar si se reduce la corrupción en el Estado. Esto sería un posible indicador de que la percepción de corrupción incide en el comportamiento fiscal tanto de quienes tienen moral tributaria como de quienes no la tienen, lo que lleva a pensar que la ausencia de moral tributaria también estaría estrechamente vinculada a la percepción de corrupción. Aquí surge un punto interesante: si se identificaran las principales razones por las cuales la gente no tiene moral tributaria o es baja, entonces posiblemente sería más claro cuál sería la solución para fortalecer la moral tributaria y al mismo tiempo se estarían solucionando aquellos problemas que incluso los que poseen moral tributaria fuerte consideran que existen en el Estado o gobierno y que por ello deciden evadir.

\footnotetext{
${ }^{1}$ Para determinar el nivel de moral tributaria (Moral Tributaria Alta, Moral Tributaria Débil y Sin Moral Tributaria) se tomó la definición realizada en Giarrizzo-Sívori, 2010.
} 
La evasión como acto de rebeldía queda de manifiesto en otro dato. Teniendo en cuenta que mejorar los ingresos es una de las causas principales en la decisión de no pagar impuestos de un individuo, una pregunta que se le hizo a la población relevada fue:

Si usted tuviera los ingresos suficientes para mantener el estándar de vida que quisiera, ¿pagaría todos sus impuestos?

Si bien el 66\% de los individuos encuestados contestaron afirmativamente, resulta llamativo que un 21,1\% contestó en forma negativa. Es decir, aún si no tuvieran problemas de ingresos afirman que 'no pagarían todos sus impuestos'. En cambio, a la pregunta Si hubiera menos corrupción en el gobierno, ¿pagaría todos sus impuestos?, solo $2,5 \%$ contesta negativamente.

Cuando se analizan las respuestas entre quienes dicen que aún si tuvieran el estándar de vida deseado 'no pagarían todos sus impuestos' $(21,1 \%$ de los encuestados), surge un dato a destacar: si bien no pagarían sus impuestos aunque tuvieran el estándar de vida deseado, el 22,4\% de ellos sí lo haría si el Estado fuera menos corrupto y otro $71,1 \%$ quizás lo haría si el Estado fuera menos corrupto.

Si tuviera el estándar de vida que desea, ¿pagaría todos sus impuestos?

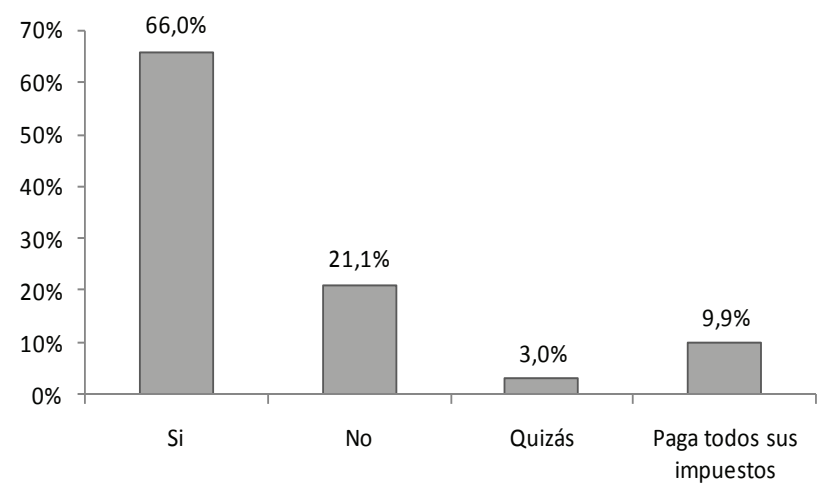

Fuente: Encuesta de Percepción Fiscal (EPF) tramo II 
Los resultados de la encuesta revelan que, el 93,5\% de los individuos que manifiestan que aún si tuvieran el estándar de vida que desean no pagarían sus impuestos, sí posiblemente lo harían si hubiera menos corrupción en el Estado. E $n$ base a estos datos se puede determinar que un 19,7\% de la población relevada (93,5\% del 21,1\%) toma el no pago como un castigo deliberado al Estado. Los denominamos 'Evasores Justicieros', esto es, individuos que aún si tuvieran el estándar de vida deseado no pagarían sus impuestos, pero sí lo harían si hubiera menos corrupción en el Estado.

De todos modos, más allá del castigo manifiesto que revelan esos individuos, no hay que perder de vista que $87,6 \%$ de los 3.300 individuos encuestados se muestran predispuestos a pagar impuestos si el Estado fuera menos corrupto. Es decir, no solo los Evasores Justicieros mejorarían su comportamiento fiscal si hubiera menos corrupción, sino que también los que evaden motivados por otras razones responderían con una mejor conducta fiscal, emulando la mayor honestidad del Estado.

\begin{tabular}{|c|c|c|c|c|c|c|}
\hline \multirow{7}{*}{$\begin{array}{l}\text { Si hubiera menos } \\
\text { corrupción en el } \\
\text { gobierno, ¿pagaría } \\
\text { todos sus impuestos? }\end{array}$} & Sí & Quizás & No & $\begin{array}{l}\text { Paga } \\
\text { todo }\end{array}$ & Total & $\begin{array}{c}\text { Población que pagaría si } \\
\text { Estado fuera menos } \\
\text { corrupto }\end{array}$ \\
\hline & $66,3 \%$ & $21,3 \%$ & $2,5 \%$ & $9,9 \%$ & $100 \%$ & $87,6 \%$ \\
\hline & 2188 & 703 & 83 & 327 & 3300 & 2891 \\
\hline & \multicolumn{5}{|c|}{ Moral Tributaria e/quienes contestaron que "SI" } & \\
\hline & \multicolumn{2}{|c|}{ Fuerte } & Débil & $\begin{array}{c}\text { Sin } \\
\text { Moral }\end{array}$ & Total & \\
\hline & \multicolumn{2}{|c|}{$81,5 \%$} & $8,7 \%$ & $9,8 \%$ & $100 \%$ & \\
\hline & \multicolumn{2}{|c|}{1783} & 190 & 214 & 2188 & \\
\hline \multirow{8}{*}{$\begin{array}{l}\text { Si tuviera ingresos } \\
\text { suficientes para } \\
\text { mantener el estándar } \\
\text { de vida deseado, } \\
\text { ¿pagaría todos sus } \\
\text { impuestos? }\end{array}$} & Sí & Quizás & No & $\begin{array}{l}\text { Paga } \\
\text { todo }\end{array}$ & Total & \\
\hline & $66,0 \%$ & $3 \%$ & $21,1 \%$ & $9,9 \%$ & $100 \%$ & \\
\hline & 2178 & 99 & 696 & 327 & 3300 & \\
\hline & \multicolumn{5}{|c|}{$\begin{array}{l}\text { Quienes contestaron que "No", ¿ pagarían si el Estado } \\
\text { fuera menos corrupto? }\end{array}$} & \\
\hline & \multicolumn{3}{|c|}{ Evasores Justicieros } & \multirow{2}{*}{ No sabe } & \multirow{2}{*}{ No } & \multirow{2}{*}{ Total } \\
\hline & Sí & Quizás & Total & & & \\
\hline & $22,4 \%$ & $71,1 \%$ & $93,5 \%$ & $5,2 \%$ & $1,3 \%$ & $100,0 \%$ \\
\hline & 156 & 495 & 651 & 36 & 9 & 687 \\
\hline
\end{tabular}

Fuente: Encuesta de Percepción Fiscal (EPF) tramo II 


\section{II.2.Ineficiencias del Estado y evasión}

La evasión como consecuencia de las ineficiencias del Estado en la administración del gasto y en la provisión de bienes y servicios públicos, es una relación muy abordada por la literatura económica. Si bien los individuos intuyen que esas ineficiencias están relacionadas con actos de corrupción que repercuten en un direccionamiento del gasto hacia sectores determinados o en una mala asignación de recursos, lo que se percibe es que los impuestos que abonan no se correlacionan con una provisión adecuada de bienes y servicios públicos. Los contribuyentes están más inclinados a cumplir la ley cuando perciben que la relación entre los impuestos pagados y los bienes y servicios obtenidos del gobierno mantienen cierta equidad $^{2}$. En función de ello, es de esperar que cualquier mejora en la eficiencia del gobierno aumente la predisposición a pagar impuestos. Así, el cumplimiento fiscal individual parece afectado por la relación entre los beneficios recibidos del gobierno (bienes públicos) y el precio pagado por ellos. Los individuos pueden sentirse estafados si los impuestos no son gastados adecuadamente. Entonces, el costo moral de evadir impuestos decrece y la moral fiscal también. En una encuesta realizada por Latinbarómetro (2008), surge que en la Argentina 26,7\% de la gente señala que evade porque los impuestos son malgastados, en tanto $32 \%$ menciona como causa la existencia de corrupción. Esas dos opciones, junto a la opción 'porque los impuestos son demasiado altos' son las que aparecen con mayor frecuencia entre las causas de por qué evaden los individuos (son mencionadas $65,6 \%$ de las veces). En la Argentina, mediciones realizadas por la UTDT revelan que solo $43 \%$ de la gente cree que el gobierno maneja eficientemente el gasto, es decir, la percepción de ineficiencia en el uso de los recursos tributarios resulta elevada.

Una pregunta que se realizó en la EPF tramo II para evaluar la relación entre esas ineficiencias y el comportamiento fiscal, fue:

Si usted viera reflejado el pago de sus impuestos en una mejora en los servicios públicos, ¿pagaría más sus impuestos?

Las categorías para responder fueron: Si; Quizás; No; No lo Sé. Como resultado, el $58 \%$ de los individuos respondieron afirmativamente, en tanto $25 \%$ señaló que 'quizás'.

\footnotetext{
${ }^{2}$ Para observar un análisis más profundo sobre este tópico ver los trabajos de Torgler 2003, 2004, 2005a, 2005b y Torgler y Martinez 2005.
} 
Si mejoraran los servicios públicos brindados por el Estado, ¿pagaría más sus impuestos?

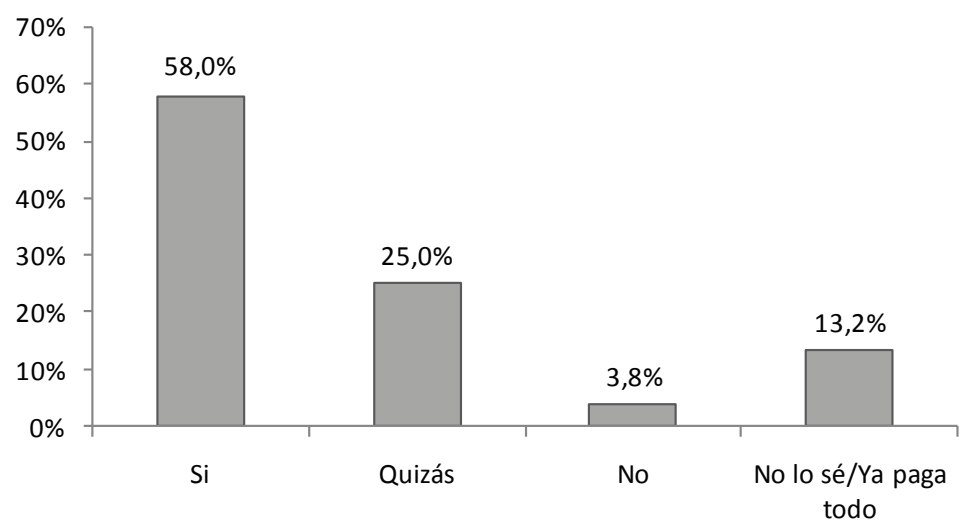

Fuente: EPF tramo II

Si se tiene en cuenta que en la pregunta sobre corrupción se hacía referencia a pagar 'todos' los impuestos y en la de eficiencia se refiere solo a pagar 'mas' impuestos, y que al mismo tiempo la gente se mostró más predispuesta a pagar si hubiera menos corrupción que si mejorara la provisión de bienes y servicios públicos, se puede inducir que la provisión de bienes y servicios parece muy vinculante al comportamiento fiscal individual, aunque en menor medida que la percepción de corrupción, que parece molestar con mayor potencia e inducir a una mayor rebelión fiscal.

\section{II.3. Injusticias y motivaciones}

Las percepciones de corrupción e ineficiencias en la provisión de servicios públicos reducen la predisposición a pagar impuestos e incrementan el descontento sobre las tasas impositivas que se pagan. Según resultados de la EPF tramo II, los individuos consideran que los impuestos en la Argentina son injustos y poco equitativos. A la pregunta: ¿Cree que los impuestos en la Argentina son justos y equitativos?, $54,7 \%$ dice que son injustos, el $36,3 \%$ señala que solo algunos impuestos son justos y equitativos. La percepción de injusticia e inequidad en el sistema tributario, es un ingrediente adicional en las decisiones vinculadas al comportamiento fiscal. 
Si hubiera una mejora en los servicios públicos brindados por el Estado, ¿pagaría más sus impuestos?

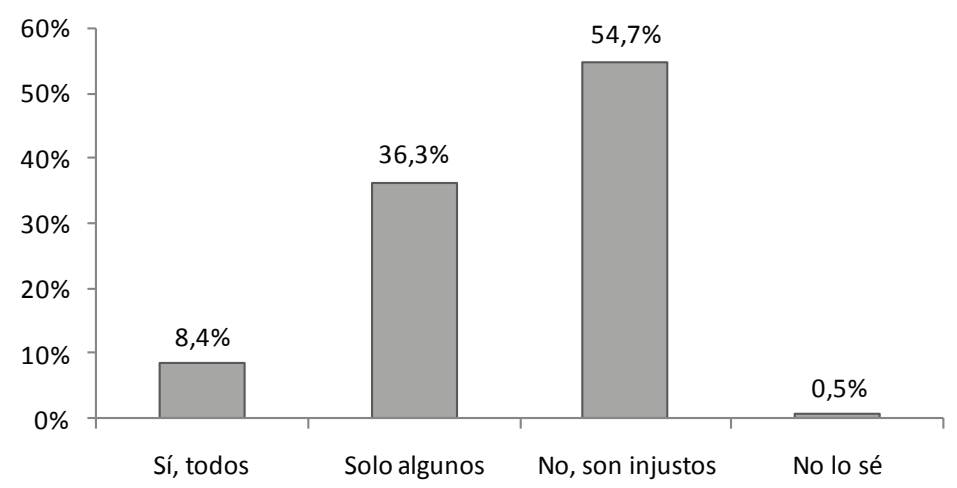

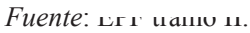

Si la percepción de tasa justa no es positiva (o lo es parcialmente) puede inducirse que el sistema tributario en sí presenta distorsiones respecto a lo que la gente espera, reduciendo la posibilidad de combatir la evasión por la vía voluntaria. Incluso esa percepción de injusticia combinada con percepciones de corrupción y de falta de eficiencia en el manejo de los recursos públicos, explicarían en buena medida por qué un segmento no menor de la población argentina no tiene moral tributaria (según la EPF tramo I el 23,4\% de la población no tiene moral tributaria en tanto otro 17,1\% tendría moral tributaria débil) y por qué casi la mitad de los individuos que sí tienen moral tributaria fuerte están dispuestos a evadir si se les presenta la oportunidad, y no sienten culpa de hacerlo (Giarrizzo-Sívori, 2010). Es de esperar, por ejemplo, que si un sujeto con moral tributaria fuerte considera que los impuestos que debe pagar no son justos, entonces opte por rebelarse y no hacerlo.

\section{CONCLUSIONES}

Los estudios sobre evasión suelen poner la atención en los contribuyentes, analizando las múltiples razones por las que evaden y simulando a partir una serie de procesos o cambios que puedan inducir el pago (más controles, castigos más severos, sistemas de cobro alternativos, etc). Esta manera de estudiar la informalidad presupone que el problema de la evasión está exclusivamente en las conductas 
de los individuos, y desentiende a esas conductas de un vínculo más global entre el gobierno y sus ciudadanos.

Sin embargo, el pago o no pago de impuestos involucra una 'relación' entre el gobierno y sus ciudadanos, que se podría categorizar como una 'relación tributaria'. Esa relación tributaria tiene características propias en cada país, que es el resultado de un vínculo permanente y dinámico, que se retroalimenta a diario, entre Estado y contribuyentes. El pago o no pago de impuestos implica una conducta o una acción del contribuyente hacia el Estado. Cuando el individuo evade, deja de pagarle al Estado, es decir, directa o indirectamente induce un daño económico sobre los recursos del Estado. La pregunta es: ¿ese daño es deliberado o no.

A lo largo de este trabajo se fue arrojando evidencia que pareciera indicar la existencia en el comportamiento tributario de algunos individuos de una especie de castigo deliberado hacia el Estado. Según la EPF al menos un 20\% de los individuos consultados no plantearon el problema de evadir como una restricción de ingresos sino como una respuesta a la desconfianza en las autoridades. Se trata del $20 \%$ de los individuos que aún si tuvieran el estándar de vida deseado no estarían dispuestos a pagar pero sí lo harían si el Estado fuera más honesto. Más allá de los valores porcentuales concretos, lo importante es resaltar que los relevamientos y trabajos de campos efectuados revelan la existencia de cierto descontento con el comportamiento fiscal del Estado y de individuos poco dispuestos a revertir o mejorar su comportamiento tributario en la medida que el Estado no haga lo mismo.

De la misma manera, puede inducirse que también la conducta de quienes evaden y no consideran que esa acción sea incorrecta (población sin moral tributaria o con moral tributaria débil) parece estar relacionada a la percepción de niveles elevados de corrupción, donde el individuo actúa emulando el ejemplo de quienes lo gobiernan. Este comportamiento se incrementa cuando la población percibe dificultades de ingresos porque adopta la evasión como una forma supervivencia y/o porque encuentra en las ineficiencias del Estado las causas de sus problemas. Así, los mismos factores que explican que evada un individuo que manifiesta contar con una moral tributaria fuerte podrían ser útiles para explicar que exista una porción elevada de personas sin 'moral tributaria'. Y esos factores tienen que ver con la percepción de corrupción sobre la clase gobernante, la percepción de ciertas ineficiencias en el manejo de los recursos públicos y la sensación de injusticia sobre las tasas que se cobran, factores cuya corrección dependen de la voluntad institucional del gobierno. 
La relación entre evasión y percepciones de injusticia, eficiencia y corrupción es muy estrecha en la economía argentina y por eso la evasión persiste, sin que esa acción sea considerada como una conducta delictiva o amoral por un importante segmento de la población. Cierto es que esto también puede derivar en que los individuos encuentren en la corrupción estatal la excusa o justificación a su comportamiento tributario incorrecto, llevando a esquemas donde el individuo evade porque persigue un fin económico, pero se auto justifica y convence de que su acción no es incorrecta al alegar que el Estado lo induce a eso. Es decir, al momento de justificar su conducta incorrecta utiliza como argumento la corrupción e ineficiencias del sistema tributario, encontrando en esos factores 'vía libre' para evadir o eludir impuestos. Evidencia de esto es que actualmente, en la Argentina, la evasión no parece incidir en la reputación social, es decir, el costo moral se encuentra cercano a cero: la sociedad no castiga el acto de evadir, y al contrario, en muchos casos la avala y se solidariza con el evasor cuando éste es penado por las autoridades.

Eso no exime al Estado de su responsabilidad en la conducta tributaria de sus ciudadanos. Cuando un individuo evade está arrojando una señal sobre su descontento con 'algo', ya sea con la forma en que el Estado administra los fondos públicos, con la forma en que se comportan los funcionarios públicos, con una relación entre impuestos pagados y provisión de bienes públicos que considera desigual $\mathrm{u}$ otros aspectos del sistema tributario general e incluso económico, como puede ser el sentirse desfavorecido o afectado por alguna política pública.

La evasión involucra una responsabilidad compartida y mutua entre gobierno y ciudadanos. Una relación que se retroalimenta y que hace difícil pensar en combatirla cuando se observan comportamientos ineficientes por parte de quienes tienen la responsabilidad de gobernar. Por eso la evasión persiste y hasta puede incrementarse en los ciclos de auge económico, desligándose del problema de ingresos.

En la medida en que se comience a evaluar la responsabilidad del Estado en la evasión, se facilitará la tarea de buscar soluciones. El objetivo de este trabajo fue justamente aportar datos para poner la mirada en la responsabilidad del Estado y cómo, a través de su comportamiento, puede estar retroalimentando la informalidad. Esto nos lleva a construir un enfoque de responsabilidad compartida entre Estrado y ciudadanos que no puede estar ajeno al diseño de políticas públicas tendientes a incrementar el pago de impuestos. 


\section{REFERENCIAS BIBLIOGRAFICAS}

Abdulai, A.G, (2009), "Political will in combating corruption in developing and transition economies: A comparative study of Singapore, Hong Kong and Ghana." Journal of Financial Crime, Vol. 16 Iss: 4, pp.387 - 417.

Ajzen, I. y Fishbein, M (1980), Understanding Attitudes and Predicting Social Behaviour, Englewood Cliffs, Prentice-Hall.

Allingham, M. y Sandmo, A., (1972), "Income Tax Evasion: A theoretical analysis." Journal of Public Economics, 1(3/4), 323-338.

Alm, J., McClelland, G., y Schulze, W., (1992), "Why Do People Pay Taxes?" Journal of Public Economics, 48(1), 21-38.

Alm, J. y Torgler, B., (2006), "Culture Differences and Tax Morale in the United States and in Europe." Journal of Economic Psychology 27, 224-246.

Corporación Latinbarómetro, Informe noviembre 2008, Chile.

Cowell, F. y Gordon, J., (1988), "Unwillingness to Pay.” Journal of Public Economics, 36(3), 305-321 Cowell, F. y Gordon, J., (1988), "Unwillingness to Pay." Journal of Public Economics, 36(3), 305-321.

Cullis, J. y Lewis, A., (1997), "Why People Pay Taxes: From a Conventional Economic Model to a Model of Social Convention." Journal of Economic Psycology, 18(2-3), 305-321.

De Soto, H., (2000), The Mystery of Capital: Why Capitalism Triumphs in the West and Fails Everywhere Else, New York, Basic Books.

Erard, B. y Feinstein, J., (1994), "The Role of Moral Sentiments and Audit Perceptions in Tax Compliance". Public Finance 49 (Suppl.): 70-89.

Erard, B. y Feinstein, J., (1994), "Honesty and Evasion in the Tax Compliance Game." RAND Journal of Economics. Vol. 25. N1.

Fatás, E. y Roig, E., (2004), "Una introducción a la metodología experimental en economía." Cuadernos de Economía 27:7-036.

Feld, L. y Frey, B., (2002a), The Tax Authority and the Taxpayer: An Exploratory Analysis, Working Paper.

Fernández, P., Clavero A. y Horrillo, M., (2008), Los enfoques de análisis de la evasión fiscal. Una revisión actual de la investigación, Universidad de Málaga.

Gamazo Chillón, J.C, (2004), "Principales trabajos empíricos en materia de evasión fiscal"; Anales de Estudios Económicos y empresariales; nº; p.p 199-217. 
Giarrizzo, V, Sivori, J. (2010). "El caso de los moralistas evasores.” Pecvnia, 10 (2010), pp. 95-124.

Hindriks, J., Keen M., and Muthoo, A., (1999), "Corruption, Extortion and Evasion," Journal of Public Economics, $\mathrm{N}^{\circ} 74$, pp. 395-430.

Índice de Percepción de la Corrupción, Transparency International (2008).

Índice de Confianza en el Gobierno, 03/2011, UTDT, Buenos Aires, Argentina.

Lederman, L., (2003), “The Interplay Between Norms and Enforcement in Tax Compliance." Ohio State Law Journal, Vol. 64.

Lewis, A., (1982), "The social psychology of taxation." British Journal of Social Psychology, 21 (2), pp. 151-158.

Mason, R y Calvin, D., (1978), “A Study of Admitted Income Tax Evasion.” Law and Society Review 13:73-89.

Mittone L., (2002) Individual Styles of Tax Evasion: an Experimental Study. Working paper n.2/2002 -CELL, Università degli Studi di Trento - CELL

Mittone L., (2002) Dynamic Behaviours in Tax Evasion. An Experimental Approach. Working Paper n.3/2002 - CELL. Università degli Studi di Trento - CELL.

Mittone L., (1997) Subjective Versus Objective Probability: Results from Seven Experiments on Fiscal Evasion. Working Paper n.4/1997 - CELL, Università degli Studi di Trento - CEEL.

Mittone L. y Bosco L., (1997) "Tax Evasion and Moral Constraints: Some Experimental Evidence.” Kyklos , v. 50, n. 3, p. 297-324.

Picur, R. y Riahi-Belkaoui, D., (2006), "The impact of bureaucracy, corruption and tax compliance." Review of Accounting and Finance, Vol. 5 Iss: 2, pp. $174-180$

Schwartz, R. y Orleans, S., (1967), “On Legal Sanctions”, University of Chicago Law Review, vol. 25, pp. 274-300.

Tanzi, V. y Zee, H.H., (2000), “Tax Policy for Emerging Markets: Developing Countries", National Tax Journal, № 53, pp. 299-322.

Torgler, B., (2003), “To Evade Taxes or not to Evade: That is The Question.” Journal of Socio-Economics, 32(3), 283-302.

Torgler, B., (2004), "Tax Morale in Asian Countries". Journal of Asian Economics, 15(2), 237- 266.

Torgler, B., (2005a), "Tax Morale in Latin America." Public Choice, 122(1-2), 133-157.

Torgler, B., (2005b)., The evolution of tax morale in Modern Spain. Georgia State 
University, Andrew Young Scholl of Policy studies.

Torgler, B. y Martinez-Vazquez, J., (2005), "The Evolution of Tax Morale in Modern Spain" International Studies Program Working Paper Series, at AYSPS, GSU paper 0521, International Studies Program, Andrew Young School of Policy Studies, Georgia State University.

Vihanto, M., (2003), "Tax Evasion and The Psychology of The Social Contract." Journal of Socio-Economics, 32(2), 111-125.

Waneryd, K.E. y Walerud, B., (1982), "Taxes and economic behavior: some interview data on tax evasion in Sweden", Journal of Economic Psycology, vol.2, pp. 187-211. 